\title{
In vitro fracture resistance of premolar teeth restored with fibre- reinforced composite posts using a single or a multi-post technique
}

\author{
Márk Fráter, DDS ${ }^{1} \star *$; András Forster, DDS ${ }^{1}$; Ádám Jantyik, DDS ${ }^{1}$; Gábor Braunitzer, DDS, PhD ${ }^{2}$; Katalin Nagy, DDS, \\ $\mathrm{PhD}^{2}$; and Simone Grandini, DDS, $\mathrm{PhD}^{3}$ \\ 1 Department of Operative and Esthetic Dentistry, Faculty of Dentistry, University of Szeged, Szeged, Hungary \\ 2 Department of Oral Surgery, Faculty of Dentistry, University of Szeged, Szeged, Hungary \\ 3 Department of Endodontics and Restorative Dentistry, Universita degli Studi di Siena, Siena, Italy
}

\section{Keywords}

bicuspid, fibreglass reinforced polymers, multipost, single post, tooth endodontically treated.

\section{Correspondence}

Dr. Márk Fráter, Faculty of Dentistry, University of Szeged, Tisza Lajos körút 64, Szeged 6722, Hungary. Email: meddentist.fm@gmail.com

doi: 10.1111/aej.12150

\begin{abstract}
The purpose of this study was to evaluate the reinforcing effect of fibre-reinforced composites (FRC) applied in premolar teeth with different techniques and minimally invasive post space preparation. Fifty extracted and endodontically treated premolar teeth were used. The teeth were divided into five groups $(n=10)$ depending on the restorative technique (Groups 1-5). Group 1: one single conventional post, Group 2: one main conventional and one collateral post, Group 3: one elastic post, Group 4: one main elastic and one collateral post, and Group 5: individual post formed of elastic posts. After cementation and core build-up, the specimens were submitted to static fracture resistance test. Fracture thresholds and fracture patterns were measured and evaluated. Group 4 showed the highest average fracture resistance among the tested groups. The multi-post techniques (group 2 and 4) exhibited statistically higher fracture resistance compared to group 1. Regarding fracture patterns, there was no statistically significant difference between the tested groups. Within the limitations of this study, the application of multiple elastic or conventional FRC posts or a single elastic post in the same root canal is beneficial in terms of fracture resistance compared to a single conventional FRC post. The elasticity or the number of posts did not influence the fracture patterns.
\end{abstract}

\section{Introduction}

Endodontically treated teeth (ETT) are structurally different from non-restored vital teeth, and they require specific restorative treatment (1). The differences include reduced moisture and dentin fracture resistance and decreased proprioception (2). According to Dietschi et al. (3), the consequences of these changes are negligible. The major issues with ETT seem to be the coronal destruction derived from caries, fractures of previous restorations, dentin loss due to the removal of the roof of the pulp chamber (4), and the weakening of the peri-cervical dentin during access preparation (5). As a result of the compromised structural integrity, an increased fracture tendency during normal function is notable (6). Thus, in most ETT, the use of intraradicular posts is rec- ommended to promote the retention of the final restoration and to biomechanically reinforce the remaining tooth structure (7). However, it has to be stated that if ETT still have substantial amount of remaining sound tooth structure, posts are not improving longevity of ETT but bear substantial risks when placing them $(8,9)$. In an attempt to address the problem of compromised structural integrity in ETT, the development and use of fibrereinforced composite (FRC) root canal posts have increased rapidly over the last 10 years (10).

Recent studies have shown that post space preparation weakens the remaining tooth structure further, thus paradoxically, the conventionally accepted process of strengthening the tooth may cause further increase in root fracture risk (11). This emphasises the importance of trying to preserve the original anatomy of the root canal 
and minimising dentin loss throughout the endo-restorative treatment. This leads to unique and irregular root canal forms in several cases, in which one FRC post cannot provide adequate mechanical friction on its own. Utilising multiple posts to treat wide, irregular endodontic cavities has already been proposed when restoring ETT (12). However, the use of this technique is limited when applying a minimally invasive approach in post space preparation.

To overcome the difficulties that irregular root canal forms pose, an elastic FRC post (everStick POST, GC Europe, Leuven) was introduced to the market in 2011 . This post is individually adaptable and its bonding and flexural properties (flexural strength: $1145 \mathrm{MPa}$, Young-modulus: $15 \mathrm{GPa}$ ) (13) appear to be superior to commercially available, prefabricated FRC posts (10).

The goal of the present in vitro study was to determine and compare the fracture resistance and fracture patterns of endodontically treated premolar teeth restored with different FRC posts in different configurations. The null hypotheses were the following: (1) The fracture resistance of the teeth restored with single or multiple posts would not be different. (2) The application of more elastic posts would not result in more favourable fracture patterns.

\section{Materials and methods}

Fifty upper premolar teeth extracted for periodontal or orthodontic reasons were selected for this study. The inclusion criteria were absence of caries or root cracks, no previous endodontic treatment, no posts or crowns, no resorption and the absence of lateral canals. Furthermore, buccolingual and mesiodistal radiographs of all teeth were taken and examined to evaluate root integrity and the number of canals present. To standardise procedures and materials, all teeth used in this study had one root canal in each root with a curvature of less than $5^{\circ}$, evaluated by Schneider's technique (14), and root length of $15 \pm 1 \mathrm{~mm}$ and rather similar mesiodistal and buccolingual dimensions $( \pm 10 \%)$ were selected.

The procedures of this study were approved by the Regional Ethics Committee for Human Medical Biological Research (University of Szeged, Hungary).

The freshly extracted teeth were immediately placed in $5.25 \% \mathrm{NaOCl}$ for $5 \mathrm{~min}$ and then stored in $0.9 \%$ saline solution at room temperature. The teeth were used within 6 months after the extraction. During specimen preparation, the soft tissue covering of the root surface was removed with hand scalers.

Before root canal treatment, all crowns were sectioned at the level of the cementoenamel junction (CEJ) perpendicular to the longitudinal axis, using a slow-speed, water-cooled diamond disc.
At the beginning of the root canal treatment, the working length was established using a direct method, by subtracting $1 \mathrm{~mm}$ from the actual root length determined by introducing a no. $10 \mathrm{~K}$-file (Maillefer-Dentsply, Ballaigues, Switzerland) until it was visible through the apical foramen. A crown down technique was used for instrumentation with Gates Glidden (Union Broach, York, PA) \#2 to \#4 drills and then the canals were instrumented using rotary ProTaper files (Dentsply, Maillefer, Ballaigues, Switzerland). The series of the ProTaper system (S1, S2, F1, F2, F3) was used for the preparation at the working length.

Irrigation was performed after every change of instrument with $2 \mathrm{ml}$ of $2.5 \% \mathrm{NaOCl}$ solution and the canal space was filled with irrigant during the instrumentation phase. A root canal lubricant (Glyde, Dentsply-Maillefer, Konstanz, Germany) was only used during the shaping of the coronal third. After shaping and cleaning, the roots were dried with $96 \%$ alcohol and paper points. Root canal filling was performed by matched-single-cone obturation with a master cone (F3 gutta-percha, Maillefer-Dentsply) matching the final instrument used for preparation and sealer (AH plus; Dentsply De Trey GmbH, Konstanz, Germany). The root access was temporarily filled with Clearfil SE Bond and Clearfil AP-X (Kuraray, Tokyo, Japan). The same composite was applied to the apical part of the root in order to prevent leakage through the apex. The teeth were then stored in an incubator (mco-18aic, Sanyo, Moriguchi, Osaka, Japan) for 1 week (at $37{ }^{\circ} \mathrm{C}, 100 \%$ relative humidity).

After 1 week of incubation, post space was prepared in the root portions of the teeth with a depth of $10 \mathrm{~mm}$, as measured from the CEJ on the buccal aspect of the tooth, but no post space preparation drill was used so that the individual anatomy could be preserved. Only the root canal filling was removed with size 3 Gates Glidden burs and ISO standard Hedstrom files, leaving a minimum apical seal of $4-6 \mathrm{~mm}$ of gutta-percha in the canal.

For the restorations, two different types of FRC posts were used: a prefabricated, conventional FRC post $(0,8$ GC Fiber Post, GC Europe, Leuven, Belgium) and an elastic FRC post (0,9 EverStick POST, GC Europe, Leuven, Belgium).

The conventional translucent FRC posts of $0.8 \mathrm{~mm}$ diameter (Fiber Post) were tried in and cleaned with alcohol afterwards. The posts did not receive any surface treatment. The elastic FRC posts were handled according to the manufacturer's instructions, with sterile tweezers. Regardless of the exact type, the main posts were placed in a way that $5.0 \mathrm{~mm}$ was left above the level of decoronation, and $10.0 \mathrm{~mm}$ was inserted into the root canal. This way, a uniform $15.0 \mathrm{~mm}$ fibre length was achieved. 
The teeth were randomly distributed in five study groups, each group consisting of 10 teeth.

Group 1 received one single conventional FRC post $(0.8 \mathrm{~mm})$. Group 2 received one main conventional FRC post and one collateral post ( $0.8 \mathrm{~mm}$ both) using a "multi-post technique". The collateral post was inserted next to the main post as apically as possible without causing manually perceivable stress but it was always deep enough to wedge the main post in the canal. Group 3 received one single elastic FRC post $(0.9 \mathrm{~mm})$. According to the manufacturer's instructions, the post was inserted into the root canal, and adapted to its form. Once adapted, the post was removed from the root canal with a needle-nose plier and light-cured for $40 \mathrm{~s}$ so that it would retain the shape of the canal. Group 4 received one main elastic FRC post and one elastic collateral post (0.9 mm both) using a multi-post technique. The collateral post was inserted next to the main post as apically as possible without causing manually perceivable stress. The posts were removed as one unit from the root canal with a needle-nose plier and then light-cured for $40 \mathrm{~s}$ maintaining their position together in the canal. Group 5 received as many elastic FRC posts $(0.9 \mathrm{~mm})$ as possible bundled according to the thickness of the root canal using the lateral condensation method described by Hatta et al. (14). These posts were gently removed as one unit with a needle-nose plier from the root canal, and then lightcured for $40 \mathrm{~s}$. It was confirmed in all cases that the elastic FRC posts were repositioned to their original position into the canal after light-curing. If resistance was met, the post surface was adjusted using carborundum point.

During the luting procedures, all groups received the same adhesive treatment by the same trained operator who completed a 3-year specialisation in restorative dentistry.

For bonding, a dual-cure one-step self-etch adhesive system (Gradia Core Self-Etching Bond, GC Europe, Leuven, Belgium) was used, according to the manufacturer's instructions. Luting of the posts and the core build-up was performed with a dual-cure resin composite core material (Gradia Core, GC Europe). Gradia Core was applied using its own automix cartridge with an 'elongation tip' for direct root canal application. After the insertion of the post(s), the composite core material was polymerised from the top of the post with an Optilux 501 quartz-tungsten-halogen light-curing unit for $60 \mathrm{~s}$ from each side (a total of $240 \mathrm{~s} /$ tooth).

In order to ensure the uniformity of the specimens, the composite resin core build-ups were standardised using cellulite core-forming matrices of the same size. These matrices were fabricated as vacuum formed foils by a dental technician modelled on a healthy premolar tooth, which was previously prepared for a crown with a $1 \mathrm{~mm}$ shoulder. The core build-up was prepolymerised for $20 \mathrm{~s}$, then glycerine gel (DeOx Gel; Ultradent Products Inc., Orange, CA, USA) was applied and final polymerisation was performed from each side for $40 \mathrm{~s}$ with an Optilux 501 quartz-tungsten-halogen light-curing unit. After the cementation procedures, the specimens were stored in physiological saline solution (isotonic saline solution $0.9 \%$ B. Braun, Melsungen, Germany) in an incubator (mco-18aic, Sanyo) for 1 week (at $37{ }^{\circ} \mathrm{C}, 100 \%$ humidity) before the fracture test. The specimens were embedded as described by Frater et al. (15).

After embedding, all specimens were immediately subjected to a fracture resistance test using a universal loading device (5848 MicroTesterl; Instron, Norwood, MA, USA). Each test was performed at a cross-head speed of $0.5 \mathrm{~mm} \mathrm{~min}^{-1}$. Load was applied at $45^{\circ}$ to the long axis of the tooth by adjusting a stainless steel ball-shaped stylus to the occlusal surface of the abutment in a stabile position (16). The failure loads were recorded in Newtons (N). A force vs. extension curve was dynamically plotted for each tooth.

After the mechanical testing, the specimens were examined for fracture patterns. A distinction was made between restorable or non-restorable fractures, following the protocol proposed by Scotti et al. (17), under optical microscope with a two-examiner agreement (Scotti et al., 2012). A restorable fracture was recognised as one above the CEJ, meaning that in case of fracture, the tooth could be re-restored, while a non-restorable fracture extends below the CEJ and extraction is likely to become necessary (18). Statistical analysis was conducted with SPSS 17.0 (SPSS Inc., Chicago, IL, USA). As the data were not normally distributed in all groups, the comparisons were performed with Kruskal-Wallis ANova with post-hoc pairwise comparisons. The level of significance was set at $P<0.05$.

\section{Results}

The mean fracture resistance $(\mathrm{N})$ and the SD for each of the five experimental conditions are presented in Figure 1. As the table shows, group 4 (containing one main and one elastic FRC post) showed the highest average fracture resistance, however, this difference was only significant compared to group 1 (containing a single conventional FRC post) $(P=0.027)$. Group 2 (containing one main and one collateral conventional FRC post) showed significantly higher fracture resistance compared to group $1(P=0.038)$. However, neither of the multipost techniques yielded significantly better results than the single elastic post technique. According to the findings, null hypothesis 1 was accepted as multiple post restorations investigated did not show significantly higher fracture resistance in all set-ups. 


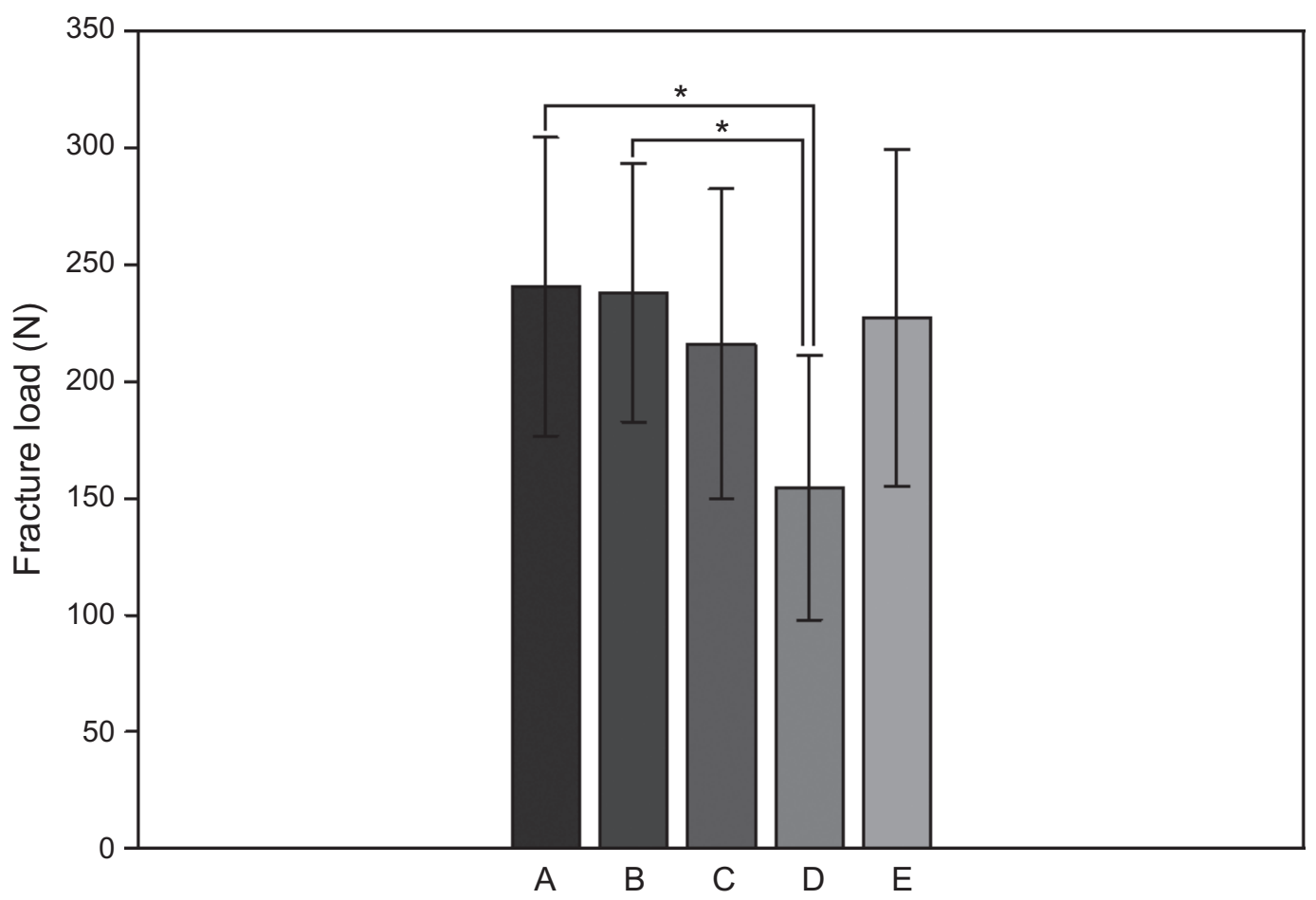

Figure 1 Fracture loads in the different study groups (mean $\pm S D$ ). A: multiple elastic posts B: multiple conventional FRC posts C: single elastic post D: single conventional FRC post E: lateral condensation with elastic posts. *Significant difference at the level $P<0.05$ (See also table).

There was no statistically significant difference when comparing the rest of the groups regarding their fracture resistance. In terms of fracture patterns, the tested groups were almost perfectly identical (see Table 1). Therefore, the null hypothesis 2, regarding fracture patterns was accepted. The application of more elastic posts would not result in more favourable fracture patterns.

\section{Discussion}

Restoration of non-vital teeth is a focal issue in restorative and prosthetic dentistry $(3,19)$, which is underlined by the fact that endodontic posts for the restoration of such teeth appeared as early as in the 1800 s. It is highly recommended that post insertion should not be carried

Table 1 Descriptive statistics of the fracture loads measured in the different groups. The values are given in Newtons

\begin{tabular}{llllll}
\hline Group & N & Mean & Minimum & Maximum & SD \\
\hline Multiple elastic posts & 10 & 240.64 & 146.88 & 330.12 & 63.85 \\
Multiple conventional & 10 & 237.99 & 165.93 & 361.63 & 55.26 \\
$\quad$ FRC posts & 10 & 215.98 & 57.22 & 312.04 & 66.33 \\
$\begin{array}{l}\text { Single elastic post } \\
\text { Single conventional FRC }\end{array}$ & 10 & 154.50 & 65.99 & 271.22 & 56.63 \\
$\quad \begin{array}{l}\text { post } \\
\text { Lateral condensation }\end{array}$ & 10 & 227.30 & 142.82 & 367.88 & 72.20 \\
$\quad$ with elastic posts & & & & & \\
\hline
\end{tabular}

out at the cost of sacrificing radicular dentin $(20,21)$. Studies have shown that post space preparation does not only weaken the tooth structure $(22,23)$ but it might also lead to cracks and defects that can concentrate stress and increase the possibility of tooth fracture and tooth loss (22). According to several authors up to $20 \%$ of ETT with radicular post suffer a vertical fracture (24-29).

It seems to be supported that dentin exhibits a fracture toughening mechanisms therefore reducing the possibility of crack progression $(4,29,30)$. For the mentioned reasons, a minimally invasive post space preparation protocol was performed. Consequentially, posts of the smallest available diameter $(0.8 \mathrm{~mm}$ GC Fiber Post; $0.9 \mathrm{~mm}$ everStick POST) were selected for the study.

This is in accordance with Sorensen et al. (31) who showed that a significant increase can be measured in the fracture resistance of restored teeth when the posts are

Table 2 The distribution of fracture patterns among the study groups

\begin{tabular}{lll}
\hline & Restorable & Non-restorable \\
\hline Multiple posts/elastic & $7(70 \%)$ & $3(30 \%)$ \\
Multiple posts/conventional & $7(70 \%)$ & $3(30 \%)$ \\
Single post/elastic & $7(70 \%)$ & $3(30 \%)$ \\
Single post/conventional & $7(70 \%)$ & $3(30 \%)$ \\
Lateral condensation/elastic & $6(60 \%)$ & $4(40 \%)$ \\
\hline
\end{tabular}


adapted closely to the canal walls. According to Maceri et al. (12), a multi-post technique may not only lead to better adaptation but will also possibly reduce pull out risk and induce restoration durability to long-term cyclic loading. Therefore, applying multiple posts in the same canal (multi-post technique) or using an individual post is aiming to achieve a better fit to the individual, preserved root canal anatomy possibly enhancing long-term clinical prognosis.

Through the use of a multi-post technique utilising small diameter posts, the operator is also able to fill large and irregular root cavities more efficiently than with a single, centrally positioned post (12).

The results of this study appear to favour the use of multiple posts in the same root canal. Both multi-post techniques (conventional FRC (group 2) and elastic FRC (group 4)) yielded significantly higher fracture resistance than the single post conventional FRC restoration (group 1). It seems supported by the findings that the elasticity of the post is not particularly important when applying a multi-post technique, as there was no statistical difference between the conventional and elastic multi-post techniques in terms of the fracture resistance of the restorations. It is interesting to note that neither of the two multi-post techniques yielded significantly different fracture resistance from the single elastic post technique. The use of a minimally invasive post space preparation therefore may explain the non-significance between the results when using a single elastic post and a multi-post technique as even a single elastic post can achieve a good fit, thus adequate stability in case of a preserved, relatively irregular root canal cross section. Therefore, the null hypothesis regarding fracture resistance was partially rejected (only for non-elastic FRC posts). A possible explanation is that the elasticity (and thus better adaptability) of the particular type of elastic post used in this study was enough to make up for the disadvantages of using a single post only. However, the limitations of this investigation cannot lead to this conclusion, so further studies on the adaptive properties of the elastic FRC posts are necessary.

The rationale behind experimenting with custom (individual) post techniques in spite of the advantages of the application of multiple posts is twofold: one of the reasons is that the problem of the insertion of geometrically uniform and symmetric single FRC post(s) into a root canal of irregularly changing diameter along its length is still not completely resolved. The other reason is that the amount of luting cement should be minimised, and the multi-post techniques, by nature, do not fully meet this requirement.

There are several methods to fabricate individual root canal posts $(1,14,32,33)$. In this study, the "lateral conden- sation method" of Hatta and colleagues was used (14). It is suggested that posts fabricated with this technique might contain a higher volume of FRC, which, in turn, may result in higher load-bearing capacity and a greater stability to the restoration. In the present study, the individual posts (group 5) yielded better results than restoration with a single FRC post (groups 1 and 3). However, the difference did not reach the level of statistical significance. These results are in agreement with those of Hatta et al. (14) and Le BellRonnlof et al. (34) in this respect.

Based on the present results, the fracture resistances yielded by the individual post technique and the two multi-post techniques were not significantly different. The reason might be the minimal invasive preparation of the root canal, which can limit the number of posts insertable to approximately the same depth thus making the cement-glass fibre ratio of the single-post techniques relatively similar to that of the multiple post techniques. This phenomena leads to the conclusion that most likely the preservation of tooth structure is the key influencing factor in term of mechanical behaviour of ETT as supported by Wandscher et al. (16).

Fracture of the post(s) or the restored tooth itself are among the most common failures of ETT restoration $(28,29)$. In the present study, no difference was found between the study groups regarding the fracture pattern. As for that matter, the results are observably uniform (see Table 2). Thus, the null hypothesis regarding fracture patterns was accepted. This contradicts the findings of Maceri et al. (12) who conclude that multi-post techniques would reduce the risk of irreversible root fractures. Nevertheless, it has to be noted that teeth examined by Maceri and co-workers have had a ferrule, and were restored with a crown making a fundamentally different set-up compared to that of the current investigation.

In the present investigation, single-root upper premolars were tested because they have been shown to be more susceptible to root fractures when submitted to occlusal loading after endodontic treatment (11). Fracture toughness tests, with their limitations, provide an indication of the load-bearing capacity of restorations in simulated clinical situations (35). To better represent a clinical scenario, the techniques used in this study could be expanded to include artificial ageing and cyclic loading experiments. The use of a crown would also achieve this aim and may change the failure patterns of the conventional FRC posts by protecting the luting agent around these posts from delamination and degradation $(36,37)$. The importance of crowns in relation to the retention of posts may also be highlighted $(38,39)$.

According to Nam et al. (2), conventional fibre posts do not improve the fracture resistance of teeth without a 
dental ferrule. This may not be the case when teeth are restored using the techniques described in this paper. There are potential clinical benefits to investigating these new restorations in further lab-based studies. The decision to not use crowns in this study was based on the observations of a number of authors where subtle differences in post behaviour and performance may be masked when teeth are definitively restored $(18,40-43)$. Despite these recommendations, the lack of a crown makes it impossible to extrapolate the results of this investigation to a clinical situation and there is a need for further experiments to bridge this gap.

The tested specimens received an oblique load (45 degree to the long axis of the tooth) which appears to be the worst-case scenario in terms of the fracture resistance of ETT as described by Wandscher et al. (16). Applying this angle of force to teeth without a dental ferrule placed significant stress on the cervical aspect of the restored tooth (44) and heavy shear forces on the post/luting agent/radicular dentine interfaces. This should represent a worst-case occlusal loading scenario for these teeth and acid-test the integrity of the tested restorations and tooth structure.

The data demonstrate low fracture loads across the samples which can be attributed to the compromised tooth structure (no ferrule), unfavourable loading forces and a lack of crown restoration. This highlights the importance of these three factors in the clinical success of restored, endodontically treated teeth and the importance of further investigations to achieve meaningful information about the best way to restore these teeth clinically.

\section{Conclusions}

Within the limitations of this in vitro study, single rooted premolars restored in the absence of ferrule show significantly higher fracture resistance when a multi-post technique is used, compared to utilising a single conventional FRC post.

No statistically significant difference was observed between using conventional or elastic FRC posts for multiple post restorations.

The teeth restored with a single elastic FRC post exhibited significantly higher fracture resistance than those restored with a single conventional FRC post.

Fracture patterns were similarly favourable in all groups. Multi-post techniques are superior to singlepost techniques in terms of achieved fracture resistance, regardless of the type of posts. Single canal teeth restored with multiple posts achieved superior fracture resistance to teeth restored with single, conventional FRC posts.

\section{References}

1. Cecchin D, de Almeida JF, Gomes BP, Zaia AA, Ferraz CC. Influence of chlorhexidine and ethanol on the bond strength and durability of the adhesion of the fiber posts to root dentin using a total etching adhesive system. J Endod 2011; 37: 1310-5.

2. Nam SH, Chang HS, Min KS, Lee Y, Cho HW, Bae JM. Effect of the number of residual walls on fracture resistances, failure patterns, and photoelasticity of simulated premolars restored with or without fiber-reinforced composite posts. J Endod 2010; 36: 297-301.

3. Dietschi D, Duc O, Krejci I, Sadan A. Biomechanical considerations for the restoration of endodontically treated teeth: a systematic review of the literature-Part 1. Composition and micro- and macrostructure alterations. Quintessence Int 2007; 38: 733-43.

4. Al-Omiri MK, Mahmoud AA, Rayyan MR, Abu-Hammad O. Fracture resistance of teeth restored with post-retained restorations: an overview. J Endod 2010; 36: 1439-49.

5. Clark D, Khademi J. Modern molar endodontic access and directed dentin conservation. Dent Clin North Am 2010; 54: 249-73.

6. Schwartz RS, Robbins JW. Post placement and restoration of endodontically treated teeth: a literature review. J Endod 2004; 30: 289-301.

7. Cecchin D, Farina AP, Guerreiro CA, Carlini-Junior B. Fracture resistance of roots prosthetically restored with intra-radicular posts of different lengths. J Oral Rehabil 2010; 37: 116-22.

8. Fokkinga WA, Kreulen CM, Bronkhorst EM, Creugers NH. Up to 17-year controlled clinical study on post-andcores and covering crowns. J Dent 2007; 35: 778-86.

9. Mangold JT, Kern M. Influence of glass-fiber posts on the fracture resistance and failure pattern of endodontically treated premolars with varying substance loss: an in vitro study. J Prosthet Dent 2011; 105: 387-93.

10. Bell AM, Lassila LV, Kangasniemi I, Vallittu PK. Bonding of fibre-reinforced composite post to root canal dentin. J Dent 2005; 33: 533-9.

11. Zicari F, Van Meerbeek B, Scotti R, Naert I. Effect of fibre post length and adhesive strategy on fracture resistance of endodontically treated teeth after fatigue loading. J Dent 2012; 40: 312-21.

12. Maceri F, Martignoni M, Vairo G. Mechanical behaviour of endodontic restorations with multiple prefabricated posts: a finite-element approach. J Biomech 2007; 40: 2386-98.

13. Lassila LV, Tanner J, Le Bell AM, Narva K, Vallittu PK. Flexural properties of fiber reinforced root canal posts. Dent Mater 2004; 20: 29-36.

14. Hatta M, Shinya A, Vallittu PK, Shinya A, Lassila LV. High volume individual fibre post versus low volume fibre post: the fracture load of the restored tooth. J Dent 2011; 39: 65-71. 
15. Frater M, Forster A, Kereszturi M, Braunitzer G, Nagy K. In vitro fracture resistance of molar teeth restored with a short fibre-reinforced composite material. J Dent 2014; 42: 1143-50.

16. Wandscher VF, Bergoli CD, Limberger IF, Ardenghi TM, Valandro LF. Preliminary results of the survival and fracture load of roots restored with intracanal posts: weakened vs nonweakened roots. Oper Dent 2014; 39: 541-55.

17. Scotti N, Coero Borga FA, Alovisi M, Rota R, Pasqualini D, Berutti E. Is fracture resistance of endodontically treated mandibular molars restored with indirect onlay composite restorations influenced by fibre post insertion? Journal of Dentistry 2012; 40: 814-20.

18. Batalha-Silva S, de Andrada MA, Maia HP, Magne P. Fatigue resistance and crack propensity of large MOD composite resin restorations: direct versus CAD/CAM inlays. Dental Materials 2013; 29: 324-31.

19. Dietschi D, Argente A, Krejci I, Mandikos M. In vitro performance of Class I and II composite restorations: a literature review on nondestructive laboratory trials-part II. Oper Dent 2013; 38: E182-200.

20. Meyenberg K. The ideal restoration of endodontically treated teeth - structural and esthetic considerations: a review of the literature and clinical guidelines for the restorative clinician. Eur J Esthet Dent 2013; 8: 238-68.

21. Paolone G, Saracinelli M, Devoto W, Putignano A. Esthetic direct restorations in endodontically treated anterior teeth. Eur J Esthet Dent 2013; 8: 44-67.

22. Bolhuis HPB, De Gee AJ, Feilzer AJ, Davidson CL. Fracture strength of different core build-up designs. Am J Dent 2001; 14: 286-90.

23. Manning KE, Yu DC, Yu HC, Kwan EW. Factors to consider for predictable post and core build-ups of endodontically treated teeth. Part I: Basic theoretical concepts. Journal 1995; 61: 685-8, 90, 93-5.

24. Fuss Z, Lustig J, Katz A, Tamse A. An evaluation of endodontically treated vertical root fractured teeth: impact of operative procedures. J Endod 2001; 27: 46-8.

25. Ferrari M, Vichi A, Garcia-Godoy F. Clinical evaluation of fiber-reinforced epoxy resin posts and cast post and cores. Am J Dent 2000; 13: 15B-8B.

26. Butz F, Lennon AM, Heydecke G, Strub JR. Survival rate and fracture strength of endodontically treated maxillary incisors with moderate defects restored with different post-and-core systems: an in vitro study. Int J Prosthodont 2001; 14: 58-64.

27. Lertchirakarn V, Palamara JE, Messer HH. Patterns of vertical root fracture: factors affecting stress distribution in the root canal. J Endod 2003; 29: 523-8.

28. Fuss Z, Lustig J, Tamse A. Prevalence of vertical root fractures in extracted endodontically treated teeth. Int Endod J 1999; 32: 283-6.
29. Tamse A, Fuss Z, Lustig J, Kaplavi J. An evaluation of endodontically treated vertically fractured teeth. J Endod 1999; 25: 506-8.

30. Kishen A, Asundi A. Experimental investigation on the role of water in the mechanical behavior of structural dentine. J Biomed Mater Res, Part A 2005; 73: 192-200.

31. Sorensen JA, Engelman MJ. Effect of post adaptation on fracture resistance of endodontically treated teeth. J Prosthet Dent 1990; 64: 419-24.

32. Eskitascioglu G, Belli S, Kalkan M. Evaluation of two post core systems using two different methods (fracture strength test and a finite elemental stress analysis). J Endod 2002; 28: 629-33.

33. Newman MP, Yaman P, Dennison J, Rafter M, Billy E. Fracture resistance of endodontically treated teeth restored with composite posts. J Prosthet Dent 2003; 89: 360-7.

34. Le Bell-Ronnlof AM, Lassila LV, Kangasniemi I, Vallittu PK. Load-bearing capacity of human incisor restored with various fiber-reinforced composite posts. Dent Mater 2011; 27: e107-15.

35. Ku CW, Park SW, Yang HS. Comparison of the fracture strengths of metal-ceramic crowns and three ceromer crowns. J Prosthet Dent 2002; 88: 170-5.

36. Mannocci F, Sherriff M, Watson TF. Three-point bending test of fiber posts. J Endod 2001; 27: 758-61.

37. Chieruzzi M, Pagano S, Pennacchi M, Lombardo G, D'Errico P, Kenny JM. Compressive and flexural behaviour of fibre reinforced endodontic posts. J Dent 2012; 40: 968-78.

38. Goracci C, Fabianelli A, Sadek FT, Papacchini F, Tay FR, Ferrari $\mathrm{M}$. The contribution of friction to the dislocation resistance of bonded fiber posts. J Endod 2005; 31: 608-12.

39. Turner $\mathrm{CH}$. Post-retained crown failure: a survey. Dent Update 1982; 9: 221, 4-6, 8-9 passim.

40. Heydecke G, Peters MC. The restoration of endodontically treated, single-rooted teeth with cast or direct posts and cores: a systematic review. J Prosthet Dent 2002; 87: 380-6.

41. Ambica K, Mahendran K, Talwar S, Verma M, Padmini G, Periasamy R. Comparative evaluation of fracture resistance under static and fatigue loading of endodontically treated teeth restored with carbon fiber posts, glass fiber posts, and an experimental dentin post system: an in vitro study. J Endod 2013; 39: 96-100.

42. Ozcan M, Valandro LF. Fracture strength of endodontically-treated teeth restored with post and cores and composite cores only. Oper Dent 2009; 34: 429-36.

43. Dietschi D, Duc O, Krejci I, Sadan A. Biomechanical considerations for the restoration of endodontically treated teeth: a systematic review of the literature, Part II (Evaluation of fatigue behavior, interfaces, and in vivo studies). Quintessence Int 2008; 39: 117-29.

44. Meira JB, Esposito CO, Quitero MF et al. Elastic modulus of posts and the risk of root fracture. Dent Traumatol 2009; 25: 394-8. 